\title{
Why are there Escalating Incidences of Confrontations in Nepali Hospitals? An Anthropological Critique
}

\author{
Kapil Dahal, $\mathrm{PhD}{ }^{*}$
}

\begin{abstract}
This article deals with the emerging phenomenon of confrontations and vandalism in hospitals in Nepal. It interrogates how far paternalism and commodification has become the feature of the Nepali health care sector and their interrelationships with each other. With the esoteric nature of medicine and different explanatory models of understanding illness episodes and healing outcomes, there is always a communication gap between the service providers and the patient party. The unfolding of the confrontation process creates space for and paves way for third party involvement in the conflict and negotiation process. The increasing confrontation also reflects falling trust between the service providers and the health seekers. This paper is based on information generated from a qualitative research carried out in two hospital settings in Kathmandu and Chitawan in different periods in 2019.
\end{abstract}

Keywords: Hospital, Conflict, Commoditization, Communication Barriers, Medicolegal Redress, Third-Party Involvement, Nepal

\section{The Onset}

The assessment of the investigation committee of the Nepal Medical Council (NMC), says that due to some "medical weaknesses" and "medical errors" that happened during the diagnosis and treatment (Mishra, 2014) at a private hospital in Thapathali, Kathmandu, Mr. Sami Risal, 47 died. Mr. Risal had been admitted to the hospital for his complaints of vomiting, headache, diarrhea, and stomachache. The NMC report mentions that though Mr. Risal had consulted the hospital to treat gastroenteritis problems, the consulting medical doctor could not recognize on time the neurological problem that he was actually suffering, which ultimately became the cause of loss of his life. The report adds that when the patient became unconscious, neither was he provided with adequate emergency care, nor was he sent for intensive care. On

\footnotetext{
${ }^{*}$ Dr Dahal has done $\mathrm{PhD}$ in Anthropology and has been working as the Lecturer in Anthropology in the Central Department of Anthropolgy, Tribhuvan University, Nepal.
} 
top of that, the patient party's requests to have a consultation by other specialists were also ignored.

The report further states that there is no clear treatment protocol at this hospital that provides specialized services. This report has also pointed out the absence of a reliable mechanism at the hospital even for the admitted patients to contact the concerned medical doctor. The lack of protocol leads to confusion about how many patients does a doctor can diagnose in a day and how much time should be given to each patient. This further complicates the situation and deteriorates the quality of consultations.

Here, the family members see that the problem lies with the physicians not to cognize the ailment on time which led to the death of the patient. The hospital argued that they tried their best for Mr. Risal's treatment. With some disputes and confrontation with the hospital, the family sued the hospital at NMC. These kinds of incidents and legal measures have become a regular phenomenon these days in Nepal. As shown in this case of Mr. Risal, it is evident that patient parties and the service providers have a differential understanding of the incident. As a backward-looking activity, both the parties describe of what had already happened. They develop a differential understanding of the incident not because of their differential "explanatory models" (Kleinman, 1980) alone but also because of their conflicting interest in an interpretation of the incident.

This article aims to highlight why such conflict, confrontations, and vandalism take place in the hospitals, which are considered to be providing generous services to the people approaching there for their health care for various kinds of ailments. In this article, I will engage with, though I cannot claim to provide definitive answer, some of the pertinent questions that an inquisitive may encounter. How the differential interpretation of the treatment outcome does lead to conflict and confrontations? Are these confrontational incidents one-time episode, or can we gaze at them in a specific pattern? Why do the patient party and the service providers cannot come up with the same view about the treatment outcome? Why does patient party come up with the decision to go for vandalism when they are not satisfied with the treatment outcome? Who are involved in such incidents? If anyone from outside their close circle involves what could be the driving factor for them to bring in there? How does it affect the negotiation process? What makes hospitals to bow down to the (dies) organized voice against them?

In shaping social relationship and social phenomena in general, whether competition is a determinant factor or it's a marginal is a matter of debate and often claimants can be divided into two poles, one side in favor of determinant and the other side who consider it as having peripheral value (Mannheim, 2003). Mannheim posits 
himself somewhere in between. Moving ahead from the debate of center or periphery, Mannheim argues that it shapes the thinking process, especially regarding the "public interpretation of reality". Individuals or groups compete with each other to establish their argument. Sometimes, such competition may manifest in the form of conflict when the competing parties can reach a consensual agreement.

Analyzing the sources of conflict Koester (1998) points out that it roots in the theoretical arena of frustration and aggression. He categorically clarified that aggression is not an instinctive drive like hunger or sex; rather, it may play an instrumental role to fulfill other purposes. It is through the social process that people learn about how to behave in conflict situations, and they are primarily shaped by the cultural realm. He proclaims that conflict is an unavoidable segment of social life, whereas one can avoid violence. Following Koester, I have critically examined the context in which Nepali health seekers and sometimes health care service providers find themselves in such an unavoidable situation and what makes them go for confrontations and the patient party goes for vandalism.

Social scientists have focused on different aspects of health research in Nepal. They have analyzed the impacts of armed conflict on the health, well-being, and mental health of the general populace (Dahal, 2018, Lucite et al. 2013, Devkota and Teijlingen, 2010). However, whether and how conflict can take place in health care setting and what kinds of consequences may lead has been largely neglected in Nepali academia. It could be mainly because commonly health care setting in the low and middle-income countries is often characterized as underserved and inadequate. Thus, even academics tend to overlook the possibility of confrontations taking place in such areas. Therefore, this article has both theoretical and applied significance (Dahal, 2020) as it analyzes respectively the behavioral aspects of the conflict and confrontations linking them with broader social processes, and it can inform the health policy and planning in Nepal about the emergence and outcome of this phenomenon.

With some background and opening remarks, the introductory part of this article orients to the whole article. In the methods section, I have briefly sketched the research design, data collection techniques, and how that information were analyzed to explore a kind of pattern presented here. Then the findings and discussions part of the article has been divided further into four different sub-sections. It mainly deals about what kinds of a situation leads to the emergence of conflict and confrontation, are there any legal provisions to address medical problems, the commoditization of health care services, and how comfortable it is to have a dialogue between the two parties and in what context third parties get involved into these hospital bases confrontations is also dealt here. 


\section{Reaearch Methods}

This article bases on qualitative information generated from fieldwork carried out in Bir Hospital, Kathmandu and College of Medical Sciences (CMS), Kathmandu University, Bharatpurat different times in 2018 and 2019. I have purposefully selected these two hospitals to have variation in terms of ownership and location of the hospital. Observation and interactions were the primary sources of information generation.

Along with these physical sites, I have also collected data from the online source of some of the selected national dailies and online media. While extracting information from online sources, I have regarded that "the online material can always be considered in the light of our offline knowledge" (Miller and Sinanan, 2017: 4). I employed this approach to recompense my inability to reach the sites, where confrontational incidents have taken place. Thus, I chose online as an ethnographic site and online materials as source of information. I have juxtaposed information acquired from different sources and explicated them to derive meaning from them instead of taking the position of apologist from either side at face value.

I carried out in-depth interviews with the service providers, including doctors working in the emergency ward and out-patient department of medicine, surgery and dentistry units, lab technicians, administrative staff, nursing staff, and private pharmacists from the respective sites. Moreover, this article also bases on information acquired from common people who have reflected upon their experiences of their encounter with doctor-patient relation. Not to disclose the identity of any category of the informants, I have used pseudonym as part of "ethnographic masking" (Murphy and Jerolmack, 2016). Data acquired from all these sources provided various themes, both the grounded and prior, to elevate them to the level of analysis and interpretation, which immensely helped to find patterns in seemingly the chaotic situation of scattered data.

\section{Vulnerability to Conflict and Vandalism}

Research participants and reported cases of vandalisms have shown that particular situation, time, people, and places have more susceptibility to violent conflict. Disputes and confrontations in various departments of the hospitals are spread asymmetrically. A service provider from Bir Hospital informed me that Emergency Ward and Intensive Care Unit (ICU) are more susceptible to differences and disputes in this hospital. He stated that:

Generally, in Nepal, we have a kind of delayed health-seeking practices. People rush at the last minute and end up at the emergency ward. Sometimes, by the time patients are brought in, they already in severe condition. When the severity escalates, then they are admitted to ICU. Occasionally, we cannot cure the patients who are provided care in the ICU. 
In addition to being a conflict prone area, emergency departments also shape certain sociality among the service providers working there. A medical doctor from CMS states that often doctors working in the emergency ward quickly lose their temperament. It is not only the 'materiality' of the emergency department that led to such sociality (Law \&Mol, 1995), but the socialness of the department also contributes to generating confrontations.

The treatment procedures of certain types of ailment can make patients go for confrontations. Dialysis patients have to wait for a long time to get their turn. Occasionally, they even do not get a chance for that. The inability to have dialysis makes them angry with the hospital and health workers, and often, they accuse the latter of not understanding their precarious health condition. They have experienced that festival time like Dashain is also confrontation prone. In these times, most of the service providers are on leave, making the patients wait for a long time, which otherwise they would have accessed swiftly. This increases the patient's dissatisfaction with the hospital and the service providers.

Service providers have also experienced that people who come to the hospital with "source-force" from their connections, within the hospital or outside of it, are the ones who ultimately end up with disputes and confrontations. By using their social capital to get admission into the hospital, they can also avoid being in a queue to get the doctor's appointment ahead of ordinary people or get access to some services. A medical officer from a hospital in Chitawan says that:

These people have a higher level of 'suggestibility'. That means, we pay more attention to their narrations and suggestions. Listening to them, excessively, sometimes makes us bypass specific procedures. In case of any problems, these people question us for not properly following the procedures.

While looking at the timing of the day, those who approach the hospital, in evening, and group, have quarrelsome tendencies. Often, these people, either the patient or their significant others, are intoxicated, and they can easily find reasons to fight with the service providers. Sitaula and Magar (2011) had shown that physical assault against health professionals in Nepal occurs mainly in the evening and night when intoxicated people come to the hospital. They mistreat, misbehave and threaten the service providers.

Generally, all the patients and their significant others are concerned about their patient; everyone thinks that his/her problem should get priority. In case of unavailability, then s/he complains against the hospital, doctor, or both for neglecting $\mathrm{him} / \mathrm{her}$. As it is related to inherent human nature, it is not possible to get rid of all these facets or to address them satisfactorily for all the patients. 
Service providers, I talked to at Bir Hospital, would like to consider those patients and their significant others, who end up having conflict, as nabujhne, the ignorant, who do not understand how the hospital and medication procedure functions. For the service providers, understanding means obeying and complying with the procedures, even if the institution itself has not developed the protocol for the treatment procedure.

\section{Are there any Legal Measures to Redress the Incident?}

There is an Act in Nepal to address assaults and attacks on health workers. The existing legal provision is Swasthya Sanstha tatha Swasthyakarmiko Surakshya Ain, 2066 B.S., Health Organizations and Health Workers Security Act (2010). Clause three of this Act states that physical attack over the health workers, gherao, disrespectful behavior, vandalism and emblazing at the health institutions are strictly prohibited. To take action against these kinds of incidents, victims have to sue at the Coordination Committee of the Ministry of Health. When the Committee receives such complaints, then it has to set up an investigation committee and whose findings direct the legal procedure. In my conversation with health workers, they think this investigation process is quite lengthy. The proposed amendment to this act in 2017 had made the doctors furious, and they closed the health care services all over the country except the emergency services. They demanded the incorporation of a 'jail without bail' provision against the culprits.

Doctors have called for a series of protests against the proposed amendments in the Act. Following the agreement between the Ministry of Health, Government of Nepal, and Nepal Medical Association, the latter withdrew all its protest programs, including the closure of hospital out-patient departments. The government agreed to incorporate their demand for a 'jail without bail' through an amendment in the existing law.

Nepali (2018) regards the law enforcement in Nepal as pessimistic mainly because of misuse or manipulation of state power through money. This has influenced up to the judiciary, and thus, for these incidents, nobody gets penalized. He further adds lack of faith in the state's law enforcement agencies and mechanisms leads to such incidents; several failed attempts are there as evidence. Therefore, there is a linkage between mounting vandalism with the increasing situation of lawlessness. Echoing with Dr. Nepali, a doctor from Bir Hospital thinks it could be because of people's lack of trust in the medico-legal redressing mechanism or people are not aware of its existence that patient party opt to go for confrontations and vandalism.

A journalist regularly reporting in this area thinks lack of adequate level of proactiveness from the side of NMC had also contributed to escalating the assault and 
violence. Regulatory authorities like NMC do not work proactively to investigate the incidents regardless of their seriousness unless there is a strong complaint. Sometimes, even the investigation procedure ends without any logical conclusions. Some of the informants, with different professions and affiliations, linked the increasing trend of assault and confrontations with existing liberal democratic space in the country. They think though democracy has not made any significant change in the livelihood of common people; however, it has liberated some defiant people to act in such way.

\section{When Health Care Services are Commodified}

The commodification of medicine and health care services is not a new phenomenon. Through their inceptions, various healing practices have adopted transactional elements to compensate the professionals are involved in healing or in producing objects and services having healing power (Dahal, 2017). On the other hand, Henderson and Petersen (2002) take commodification of health care as a new social realm embedded with health policy shifts such as the deregulation and privatization of services. No one, however, can repudiate that this practice has contributed to making health as a 'commodity' and the individual subject of health care as 'client' or health care 'consumers'.

Nepali print media often present money-making as a prime factor leading to confrontations in health institutions. This also makes hospitals not make referrals (Annapurna Post, 2016) even if they can treat the patient. Budhathoki (2011), a doctor by profession, has observed that mounting urban-centric, money sloping, oblivious, and inept doctors are problematic in this regard. He thinks it has made pharmaceutical companies launch products in the market with "gift "and commissions for the doctors. Ultimately the health seekers have to pay for these medical gifts (Brhlikova et al., 2011), leading to more prescription of avoidable antibiotic and costly prescriptions, creating antibiotic resistance and fetching in superfluous diseases.

People salaried with the government hospitals think that commodification of health care services has embraced only at the private hospitals. An administrative officer from Bir Hospital says, "the confrontational problems emerging at the government hospitals are not because of the monetary matters. Patients can even get free medication here". However, while looking at the news from government hospitals and gazing at the ground reality in the field, it becomes apparent that commodification of medication has encroached primarily from the side of the prescription of unnecessary medicines (Dahal, 2017). While looking at the long queue of medical representatives of private pharmaceuticals in hospitals, one can easily see that commodification can flourish from any hospitals. Lack of trust among various actors present in the public 
health sector in Nepal and India makes trust and mistrust personalized and continually suspect (Brhlikova et al. 2011).

When diagnosis and treatment of a patient entangle with its cost, then doctors feel that they have to adapt the treatment trajectory appropriate for the specific condition. When the affordability of the patient is prioritized instead of his/her pathological condition to decide the appropriateness of the treatment trajectory, this eventually harms efficacy and ethics of medicine.

\section{Barriers in Physician-Patient Communication}

One of the factors that frequently causes discomfort to the patient party is how service providers communicate with them. As shown initially in the case of Sami Risal, NMC's investigation has pointed out the rudeness of doctors in their interactions with the patient party. While inquiring about the condition of patients, one of the doctors responded by saying-“are you a doctor or me?" The doctors failed to show sympathetic behaviors towards Risal's family and relatives even when his situation began to deteriorate. Moreover, neither they let the patient to be checked by other specialists, nor was he allowed to be taken to another hospital.

A medical doctor from Bir Hospital has realized that doctors have to wholly and clearly converse with the patients or their relatives about the patient's situation. In the pretext of compromise on such procedure, if whatsoever unpleasant occurs, then that can aggravate the situation. The differential understanding of ailment conditions, if any, by the patients and the service providers hinders communication between the two sides. Mr. Risal's case is an impeccable illustration of this form of the negligence of patients' ideas and views.

Patient satisfaction depends upon both ways of medically handling the ailment and communicating with the patient and/or their significant others about what is going on and the rationale behind that (Dahal, 2017). Unfriendly behaviors and deficiency of transparency in a communication process about the medication procedure often increases the complexities in doctor-patient relation and negatively affects patients' behavior towards the hospital. Dahal (2019) has pointed out that the communication gap between the service providers and health seekers is one of the critical factors that have makes people living closer to the border areas look for health care services across the border in Indian towns and cities.

In his effort to save the face of doctors in communication with the patient parties, a medical doctor at CMS thinks that communication problem lies mainly with the paramedic and other administrative staff, not with the medical doctors:

In different phases of our academic training up to the MD level, we learn how to communicate effectively with the patients and their relatives. Our junior staff do 
not have such an opportunity to learn. Therefore, sometimes, problems emerge because of their weakness in communications.

\section{Involvement in Conflict and Mediation Process}

Nepal Medical Council's report on Mr. Shankar Rimal's" incident at a private hospital based in Sitapaila area has clearly showed that there were some weaknesses from the side of the patient party. The report indeed stated that earlier Mr. Rimal and his family members were contented with the negotiation process and compensation, and only then he was discharged from the hospital. Later on, they came to the hospital with added demands and new people demolished the hospital to build pressure.

What Trishna K. C. has said regarding how she became able to acquire compensation also acknowledges the involvement of third parties, different kinds of institutions and individuals, in the compensation process. She stated, "I am indebted to (mass) media and rights activists for their support, which made me entitled to the compensation after eleven years of the incident". Trishna acquired Rs. 6,17,119.00 (Rs. Six Lakha Seventeen Thousands and One Hundred Nineteen) as compensation for the multiple disabilities that she got from the hospital for wrongful treatment of her left eye (Kunwar, 2011).

Comprehensive news coverage on vandalism and confrontation at hospitals by Annapurna Post (2016) has elaborated on the active involvement of Giroha, the gang, which projects every death at a hospital as a doctor's carelessness. These people go to the street with the muscle power and demand for the compensations. Hospital management teams are afraid of disclosing their doings. Some of the hospitals offer compensation to them, even without committing any gaffes.

In response to such Giroha, Dr. Sharma from Chitawan has observed that these days, each hospital has kept a group of men to give protection to the hospital in times of need. As he stated:

While serving at a hospital in Kathmandu in my initial years, once, I have met such group of people. I got to know accidentally when one of them was admitted for his stomachache. He denied providing deposit at the time of admission. The diagnosis did not show any problem in his stomach. Later on, after his discharge, we got to know from our administrative officer that he was admitted to his protection from another gang. Later on, we began to identify them as palekoketa, the boys kept by the hospital, and behave differently.

Taking the help of an organized third party seems to be emerging as a new norm in the health care domain. Both the patient parties and service providers/hospitals have begun

** A case registered (R.N. 488) at NMC on July 9, 2017. 
to take such support, though, for different purposes. This is an indication and reflection of the lack of implementations of legal provisions or declining trust towards the law enforcement mechanisms and actors involved in warrantying primary health in Nepal (Brhlikova et al., 2011). This can encumber as a somber threat in the health care sphere of Nepal.

\section{Concluding Remarks}

Confrontation has become an inevitable concomitant outcome of service providers-patient interactions in Nepal. It primarily begins with health service providers' and families' different views on what constitute good care. It also reflects the manifestations of grievances and agonies of the people. When there is a loss of life, health, or someone acquires deformity, then, it is paralleled by a physical attack on the individuals and institutions involved in such incidents. The above discussions show that the inability of media and general people to see the differential role of the hospital, doctor, lab, and other service providers makes them solely blame doctors for any failures or any dispute that emerges at the hospital. Prevalence of confrontations both in the private and also in the government hospital shows the severity of the matter.

With their variant explanatory models, the patient party and the physicians come up with a differential interpretation of the outcome of the treatment episode. In case of extreme unwanted situations such as death or deformity of the patient's body or body part(s), both sides often clash with divergent opinions. Each side attempts to establish the supremacy of its own "interpretation of the reality" (Mannheim, 2003). With differing interest, motives, priorities and knowledge base, makes them unable to come up with a consensus to view the episode of the medical outcome on which they are debating. This sometimes leads to the situation of violent conflict and confrontation at the hospital.

The mounting tendency of hostilities between the two sides signify the putrefying medical paternalism (Crichton, 2017), which used to prevail for a long time in the Nepali health care domain. It also implies that people are becoming aware of and their knowledge about the appropriate way of administering health care services is also increasing. Commoners are claiming through the performance of confrontations that they are no more ignorant. Health care services in Nepal is entering into the commercialized domain and as a consumer of health care commodity people have become more concerned and conscious about what is being done in their health and body. On the other hand, it has also become both the cause and effect of falling trust between the service providers and the health seekers and eventually leading to the decline of paternalism in the field of medicine. 
The esoteric nature of medicine and the medical profession leads to a communication gap between the service providers and the patient party. Both sides have their explanatory models, which often lead to differences in understanding the incident. People blame doctors for using their bodies as labs to make a series of medical experiments. Whereas, doctors' rationale is that medicine is not mathematical science where they cannot find a hundred percent accuracy, and thus, they have to make continuous efforts to help the patients get recovered. On top of this, in the Nepali context, there is always a problem of who is the appropriate person to get information from the doctor; is it the patient himself/herself or his/her family members?

The very social capital that privileges the health seekers to bypass the rules such as to be in the queue for the service may erupt as the source of misery. Due to their higher degree of suggestibility, sometimes medics do not follow the diagnosis protocol for the swiftness or bring down the treatment cost. In the case of the unwanted outcome, the patient party blames the service providers for not properly following the treatment protocol. This kind of situation can be regarded as the dark side of social capital (Villalonga-Olives and Kawachi, 2017, Campos-Matos, Subramanian, and Kawachi, 2016).

It is not only the family members and relatives who are involved in confrontations but also some other people who are not directly related to patients. Their participation in protest for the stranger patients is also linked with biosociality emanating from the shared vulnerability of, what they regard as, medical negligence. It would have been fair had their involvement was shaped by the shared vulnerability. Instead, many service providers would like to consider that their behaviors seem to have been mediated by the monetary transactions with the patient party. Taking the help of an organized third party appears to be emerging as a new reality in the Nepali health care domain by both the patient parties and service providers. This is an indication and reflection of poor implementations of legal provisions and declining trust towards law enforcement mechanisms of the country.

\section{Acknowledgements}

I am very much thankful to the University Grant Commission (UGC), Nepal for providing the research grant to conduct this ethnographic study. I am also thankful to all the research participants from both the field sites, Bir Hospital, Kathmandu and College of Medical Sciences, Bharatpur, Chitawan. I am equally indebted to all the media, reporters and authors whose articles and reporting have been frequently cited and consulted in developing this article. 


\section{References}

Annapurna Post. (2016). Upacharma Laparbahi. (Text in Nepali- Negligence in the treatment).Annapurna Post, 12/10/2016, Kathmandu, Nepal.

Brhlikova et al. (2011).Trust and the regulation of pharmaceuticals: South Asia in a globalized world. Globalization and Health 2011 7:10.

Budhathoki, P. (2011). Chikitsak Pani Sudhrinu Parchha (Text in Nepali- Doctors need to correct themselves). Kantipur, 2011-05-11. Kathmandu, Nepal.

Campos-Matos, I., Subramanian, S. V., \&Kawachi, I. (2016). The 'dark side' of social capital: Trust and self-rated health in European countries. The European Journal of Public Health, 26(1), 90-95.https://doi.org/10.1093/eurpub/ckv089.

Dahal, K. B. (2020). Understanding confrontations between the health service providers and the health seekers in Nepal: a medical anthropological Study. A research report submitted to the University Grant Commission, Nepal.

Dahal, K. B. (2019). Cross border medical travel to India: an anthropological exploration of Lakhanpur area of Nepal's Tarai/Madhesh. Nepali Journal of Contemporary Studies, 14(1), 47-68.

Dahal, K. B. (2018).Conflict, Gender and Body Politic in Nepal: Anthropological Engagement with the Threatened Lives and Well-Being of Women. Washington, DC: Academica Press.

Dahal, K. B. (2017). Anthropology of health seeking practices and consultation with service providers: A study among the women in Nepal's Tarai. Ph.D. dissertation submitted to Faculty of Humanities and Social Sciences (FOHSS), Tribhuvan University(TU), Kathmandu, Nepal.

Devkota, B., and van Teijlingen, E.R. (2010). Understanding effects of armed conflict on health outcomes: the case of Nepal. Conflict and Health, 4 (20).https://doi.org/10.1186/1752-1505-4-20

Henderson, S. \& Petersen, A. (2002). Introduction: Consumerism in health care. In S. Henderson, \& A. Petersen. (Eds.), Consuming health: Commodification of health care (pp.1-10). Routledge.

Kleinman, A. (1980). Patients and healers in the context of culture: An exploration of the borderland between anthropology, medicine, and psychiatry. University of California Press.

Koester, O. (1998). Cultural variation in conflict resolution: alternatives to violence. International Journal of Conflict Management, 9(4), 380-384.

Kunwar, S. (2011).GalatUpacharkoKshyatipurti (Text in Nepali- Compensation for the wrong treatment). Kantipur, 2/3/2011, Kathmandu, Nepal.

Law, J. \&Mol, A. (1995).Notes on materiality and sociality.The Sociological Review, 43, 274-294.https://doi.org/10.1111/j.1467-954X.1995.tb00604.x. 
Luitel, N. P., Jordans, M. J., Sapkota, R. P., Tol, W. A., Kohrt, B. A., Thapa, S. B., ... and Sharma, B. (2013). Conflict and mental health: a cross-sectional epidemiological study in Nepal. Social psychiatry and psychiatric epidemiology, 48(2), 183-193.

Mannheim, K. (2003).Competition as a cultural phenomenon. In G. Delanty and P. Strydom (Eds.), Philosophies of social science: The classic and contemporary readings (Pp. 128-133). Philadelphia: Open University Press.

Miller, D. and Sinanan, J. (2017).Visualisingfacebook: A comparative perspective.UCL Press.

Mishra, A. (2014). NorvicAspatalleBiramikoUpacharPrakriyamaTrutiGarekoThahar (Text in Nepali- Norvic hospital committed error in the treatment process). Kantipur, 10/14/2014, Kathmandu, Nepal.

Murphy, A., \&Jerolmack, C. (2016).Ethnographic masking in an era of data transparency. Contexts, 15(2), 14-17.DOI: 10.2307/26370371.

Nepali, P. (2018). Doctor le abaafnaitaukojogaunekiupachargarne? (Text in NepaliWhether doctors have to save them or treat the patients?). http://swasthyakhabar.com/news-details/21371/2018-02-22.

Crichton, P. (2017). Paternalism v. autonomy. The British Journal of Psychiatry, 210(1), 83-83. DOI: https://doi.org/10.1192/bjp.210.1.83a.

Sitaula, S., \&Magar, A. (2011). Medical practice in the peripheral health centers in Nepal. Journal of Nepal Health Research Council, 9(19), 198-200.

Villalonga-Olives, E., and Kawachi, I. (2017). The dark side of social capital: A systematic review of the negative health effects of social capital. Social Science \& Medicine, 194,105-127. https://doi.org/10.1016/j.socscimed.2017.10.020. 